\title{
Escalas clínicas para evaluar el envejecimiento cutáneo: una revisión de la literatura
}

\section{Clinical scales for assessing skin aging: a review of the literature}

\author{
Viridiana Vera Ramírez, ${ }^{*}$ Martha Alejandra Morales Sánchez, ${ }^{\ddagger}$ \\ Fermín Jurado-Santa Cruz, ${ }^{\S}$ Armando Medina Bojórquez
}

\section{RESUMEN}

En el presente trabajo se realizó una revisión actualizada de las diferentes escalas clínicas que se utilizan para evaluar el envejecimiento cutáneo, proceso que es tomado con poca relevancia en el ámbito médico, pero que impacta de manera importante en la calidad de vida de cada paciente, además de ser uno de los principales factores que incrementan la susceptibilidad para el desarrollo de cáncer de piel. Por lo anteriormente descrito, sugerimos realizar una detección precisa del envejecimiento cutáneo de manera individual para proporcionar el tratamiento adecuado, mejorar el pronóstico y reducir su avance o complicaciones. Ese objetivo en gran parte está limitado por el uso de escalas que no permiten una evaluación de la respuesta terapéutica en la práctica clínica habitual, además de la calificación subjetiva emitida por el médico y el paciente. El propósito de este trabajo fue llevar a cabo una revisión de la literatura existente sobre las escalas clínicas que se utilizan para la evaluación del envejecimiento cutáneo, iniciando con la descripción del mismo, sus características e importancia clínica. Posteriormente, se describen las diferentes escalas diagnósticas y de evaluación de la respuesta terapéutica, con lo cual se puede realizar un análisis integral; se señala la utilidad del empleo de la dermatoscopía y su aporte en dicha evaluación como método complementario en la valoración ya mencionada, y al final se propone un algoritmo de evaluación en la población mexicana.

Palabras clave: Envejecimiento cutáneo, dermatoscopía, escalas de medición.

\section{ABSTRACT}

The present work is a current review of the different clinical scales used to evaluate skin aging, a process that is taken with little relevance in the medical field, but that has a significant impact on the quality of life of each patient, besides being one of the main factors that increase the susceptibility for the development of skin cancer. Due to the above described, we suggest to carry out an accurate detection of skin aging individually, to provide the appropriate treatment, improve the prognosis of the pathology, and reduce its progression or complications. This objective is largely limited by the use of scales that do not allow an evaluation of the therapeutic response in routine clinical practice, in addition to the subjective rating issued by the physician and the patient. The objective of this work was to carry out a review of the existing literature on the clinical scales used for the evaluation of skin aging, beginning with its description, its characteristics and clinical importance. Subsequently, the different diagnostic and evaluation scales of the therapeutic response are described, with which a comprehensive analysis can be carried out. The usefulness of the use of dermoscopy and its contribution to said evaluation as a complementary method in the aforementioned assessment is pointed out, and at the end, an evaluation algorithm is proposed in the Mexican population.

Keywords: Skin aging, dermoscopy, measurement scales.

\section{INTRODUCCIÓN}

El fotoenvejecimiento es el principal factor asociado al desarrollo de tumores malignos a nivel cutáneo, debi-

\footnotetext{
* Dermatóloga.

‡ Jefe de Enseñanza y asesora de tesis.

$\S$ Responsable del Centro.

"Subdirector de Atención Médica.
}

Centro Dermatológico «Dr. Ladislao de la Pascua», SSCDMX do principalmente a la exposición solar crónica. ${ }^{1}$ En la actualidad, se han desarrollado un poco más de 100 escalas para evaluar el envejecimiento cutáneo, ${ }^{2}$ con la finalidad de contar con un instrumento replicable para analizar de manera fidedigna y precisa el envejecimiento y fotoenvejecimiento cutáneo, y que a su vez permita

Citar como: Vera RV, Morales SMA, Jurado-Santa Cruz F, Medina BA. Escalas clínicas para evaluar el envejecimiento cutáneo: una revisión de la literatura. Rev Cent Dermatol Pascua. 2021; 30 (2): 68-75. https://dx.doi.org/10.35366/101176 
proporcionar a los pacientes pautas de tratamiento para mejorar su calidad de vida. A pesar de lo antes descrito, no se ha propuesto ninguna escala específica que pueda ser ampliamente utilizada por los diferentes especialistas, por lo que es imprescindible establecer instrumentos de evaluación clínica que permitan analizar de manera sistematizada y válida el envejecimiento cutáneo y la respuesta terapéutica acorde a cada caso.

\section{Envejecimiento y fotoenvejecimiento cutáneo}

El envejecimiento de la piel es un proceso determinado por el declive de las funciones celulares, secundario a daño molecular, como resultado de la exposición a factores intrínsecos y extrínsecos ambientales a lo largo de la vida de una persona. Los datos clínicos característicos del envejecimiento son la presencia de arrugas finas y gruesas, pérdida de la elasticidad de la piel, disminución del volumen del tejido graso y discromías. El envejecimiento cutáneo se divide en dos tipos: el intrínseco y el derivado de factores ambientales (extrínseco) también llamado fotoenvejecimiento ocasionado por la exposición crónica a la radiación ultravioleta, siendo el segundo el más común en las poblaciones actuales.

\section{Mecanismos intrínsecos y extrínsecos del envejecimiento cutáneo}

El mecanismo intrínseco se relaciona con factores genéticos. Se sabe que la principal alteración es el acortamiento de las secuencias repetitivas de ADN no codificantes al final de los cromosomas (telómeros), que se acortan tras cada ciclo celular hasta que se alcanza una longitud crítica, y el ciclo se detiene o se produce la apoptosis celular. Los datos clínicos característicos del envejecimiento intrínseco incluyen la presencia de arrugas, la apariencia laxa y la pérdida de volumen, sobre todo de tejido adiposo.

El envejecimiento extrínseco es ocasionado, en la mayoría de los casos, por la exposición crónica a los rayos ultravioleta (UV) que inducen mutaciones en el ADN por la generación de especies reactivas de oxígeno, promoción de la melanogénesis y degradación de la matriz extracelular por la producción de metaloproteinasas (MMP-1, MMP-3, MMP-9), además de disminución de la neocolagénesis. ${ }^{3}$ Otros factores inherentes al huésped, como el género, etnia, raza, tabaquismo, tipo de alimentación, privación del sueño, estrés y uso de cosméticos ocasionan daño oxidativo al ADN e incrementan el reclutamiento de células inflamatorias a través de los cuales la piel envejece en forma prematura. ${ }^{4} \mathrm{El}$ fotoenvejecimiento afecta las áreas expuestas al sol y se caracteriza clínicamente por el desarrollo de alteraciones pigmentarias (lentigos solares, efélides, manchas hiperpigmentadas y coloraciones amarillentas no características del tipo de piel), arrugas finas y gruesas, resequedad, laxitud de la piel $\mathrm{y}$ telangectasias. ${ }^{3}$

Otros datos clínicos menos prevalentes y asociados al envejecimiento de la piel incluyen arrugas finas entrecruzadas, presencia de pliegues nasolabiales, bolsas infraorbitarias, ptosis palpebral, ojeras, cutis rhomboidalis nuchae, hipomelanosis guttata, quistes de milium, hiperplasias sebáceas, comedones, síndrome de Favre-Racouchot, xerosis, queratosis actínicas y carcinoma espinocelular, basocelular y melanoma. ${ }^{2,5}$ Este último es el tumor cutáneo maligno más agresivo. Cada año se diagnostican aproximadamente 132,000 casos, lo anterior con base en los datos proporcionados por la Organización Mundial de la Salud. En México se ha observado incremento en su incidencia de aproximadamente $500 \%$ en los últimos años, según las estadísticas publicadas por la Clínica de Melanoma del Instituto Nacional de Cancerología. El tipo de melanoma acral lentiginoso es el más frecuente en la población mexicana, en quienes prevalecen los fototipos cutáneos III y IV.

Recientemente una nueva propuesta plantea considerar las características fenotípicas de la piel de los individuos (atrófica e hipertrófica) como parte de la clasificación del envejecimiento. Patologías dermatológicas como las queratosis actínicas (lesiones precancerosas) asociadas al desarrollo de carcinoma epidermoide se manifiestan en la piel de individuos con fenotipo atrófico, independientemente del predominio del envejecimiento intrínseco o extrínseco que presenten. ${ }^{6}$

\section{Escalas clínicas para evaluar el envejecimiento de la piel}

La escala ideal debe poder evaluar el envejecimiento de la piel de manera simple, ser de fácil aplicación, no invasiva, objetiva, sistematizada y que permita, además, ser utilizada para realizar diferentes estudios clínicos y epidemiológicos. De las más de cien escalas ${ }^{2}$ desarrolladas en la actualidad, en la Tabla 1 se describen las principales, las más empleadas en la práctica clínica habitual, ${ }^{7,8}$ aquellas ${ }^{9-13}$ con excelente validez de contenido, estructura interna y confiabilidad, de acuerdo con los estándares basados en el consenso para la selección 
Tabla 1: Principales datos clínicos evaluados por las escalas de envejecimiento y fotoenvejecimiento cutáneo.

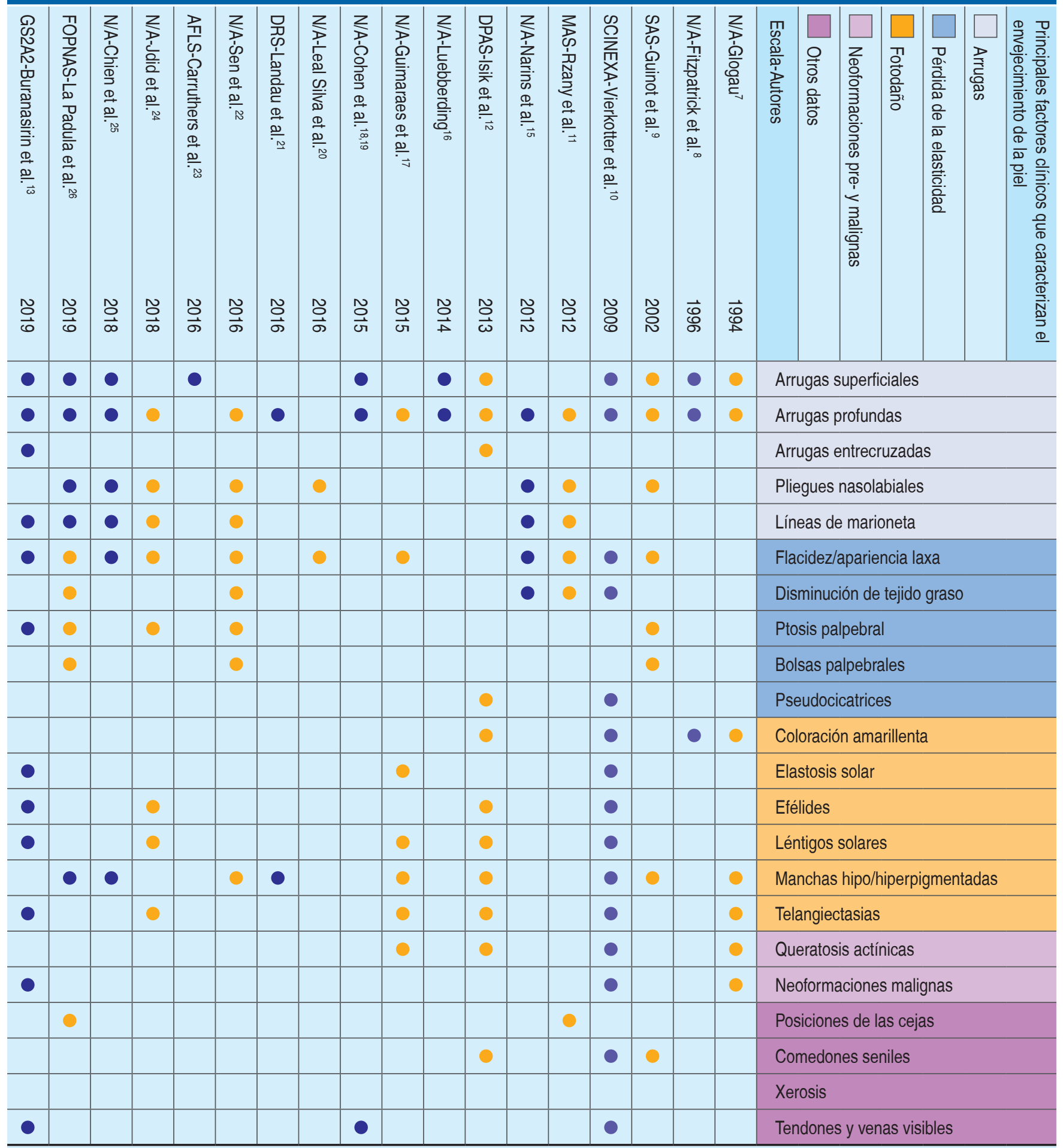

SAS = Skin Aging Score, SCINEXA = SCore of INtrinsic and EXtrinsic skin Aging, MAS = Merz Aesthetics Scale, DPAS = Dermoscopic Photoaging Scale, DRS = Décolleté Raiting Scales, AFLS = the Allergan Fine Lines Scale, FOPNAS = the new Face Objective Photo-Numerical Assessment Scale, GS2A2 = Global Subjective Skin Aging Assessment. 
de instrumentos de medición COSMIN (por sus siglas en inglés) ${ }^{14}$ y algunas otras recientemente descritas que evalúan zonas específicas. ${ }^{15-26}$

La principal región anatómica que toman en cuenta estas escalas se concentra en la cara, dividiéndola para su análisis en tercio superior, ${ }^{20,27}$ tercio medio ${ }^{20,28,29}$ y tercio inferior. ${ }^{15,20}$ Un pequeño número de escalas se limitan al cuello, ${ }^{30}$ tórax anterior ${ }^{23,31}$ y extremidades superiores. ${ }^{17,19,25,32}$ La Figura 1 muestra las principales zonas evaluadas en cara por las diferentes escalas clínicas.

Las características de la población empleadas para el desarrollo y validación de algunas de las principales escalas de envejecimiento y fotoenvejecimiento de la piel, así como su uso clínico se resumen en la Tabla 2.

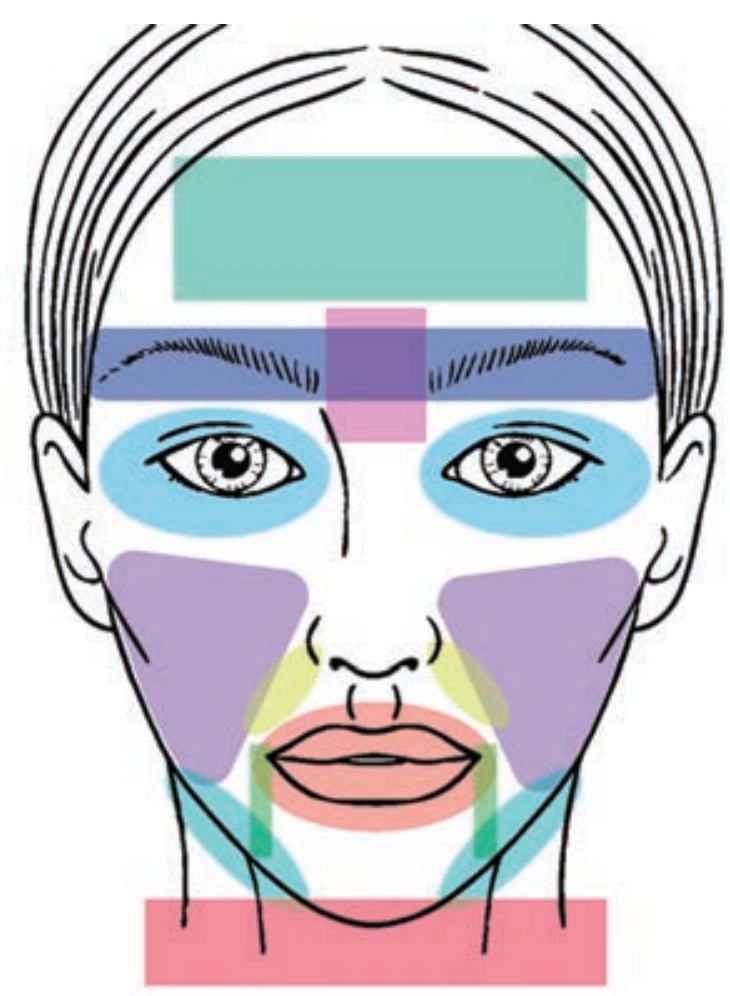

Frente

Región glabelar

Cejas

Párpados y «patas de gallo»

Mejillas

Figura 1: Principales regiones evaluadas en cara por las diferentes escalas de envejecimiento y fotoenvejecimiento.
Las escalas de severidad de Glogau ${ }^{7}$ y arrugas de Fitzpatrick ${ }^{8}$ son ampliamente conocidas y generalmente utilizadas en la práctica clínica habitual para el diagnóstico del fotoenvejecimiento. Ambas son de rápida y fácil aplicación, analizan de manera subjetiva, y clasifican el envejecimiento y fotoenvejecimiento en cuatro y tres grados de severidad, respectivamente, dependiendo de la edad cronológica de los individuos, por lo que tienen la limitación de no poder ser usadas para calificar la respuesta terapéutica de manera objetiva y cuantificable como las escalas DPAS, ${ }^{12}$ GS $2 A 2,{ }^{13}$ SAS,${ }^{9}$ SCINEXA ${ }^{10}$ y MAS, ${ }^{11}$ las cuales permiten evaluar teniendo en consideración un puntaje basado en la severidad de los datos clínicos esperados para cada rango de edad.

SCINEXA ${ }^{10}$ es la única escala diagnóstica que contiene casi la totalidad de datos clínicos asociados al envejecimiento. Su empleo permite discriminar entre los factores intrínsecos y extrínsecos predominantes en la etiología del envejecimiento, para así poder indicar las medidas terapéuticas específicas, según el tipo de envejecimiento. Sin embargo, compromete áreas no fotoexpuestas como espalda y cara posterior de antebrazos, lo que requiere mayor tiempo para su llenado y la necesidad de que solo especialistas capacitados sean los que las utilicen, lo cual dificulta su uso en la práctica clínica habitual.

La escala DPAS ${ }^{12}$ emplea la dermatoscopía para su análisis y determina con rango de 11 a 44 puntos el envejecimiento basado en la observación de tres regiones de la cara: frente, mentón y región malar derecha e izquierda, lo que también permite realizar una evaluación de la terapéutica empleada antes y después de su aplicación de manera objetiva, con la única limitante de que la unidad médica donde se desarrolle el estudio debe contar forzosamente con un dermatoscopio.

La escala subjetiva de valoración global del envejecimiento de la piel ${ }^{13}$ (GS2A2, por sus siglas en inglés) es la primera escala elaborada a partir de la opinión de 145 dermatólogos certificados. Para su llenado se requiere observar tres signos: atrofia, discromías y la presencia de lesiones malignas. Utilizar una escala tipo Likert para su medición, permite cuantificar la respuesta terapéutica con un puntaje total que oscila entre 18 y 90 puntos.

La escala estética de Merz ${ }^{11}$ (MAS, por sus siglas en inglés) examina particularmente las características de las arrugas, tanto dinámicas como estáticas, y toma en cuenta la posición de las cejas, la región del párpado inferior y el volumen del cuello, áreas que no son consideradas en otras escalas. Su análisis por tercios de la 
Tabla 2: Principales escalas con validez de contenido, estructura interna y adecuadas pruebas de hipótesis que evalúan el envejecimiento cutáneo.

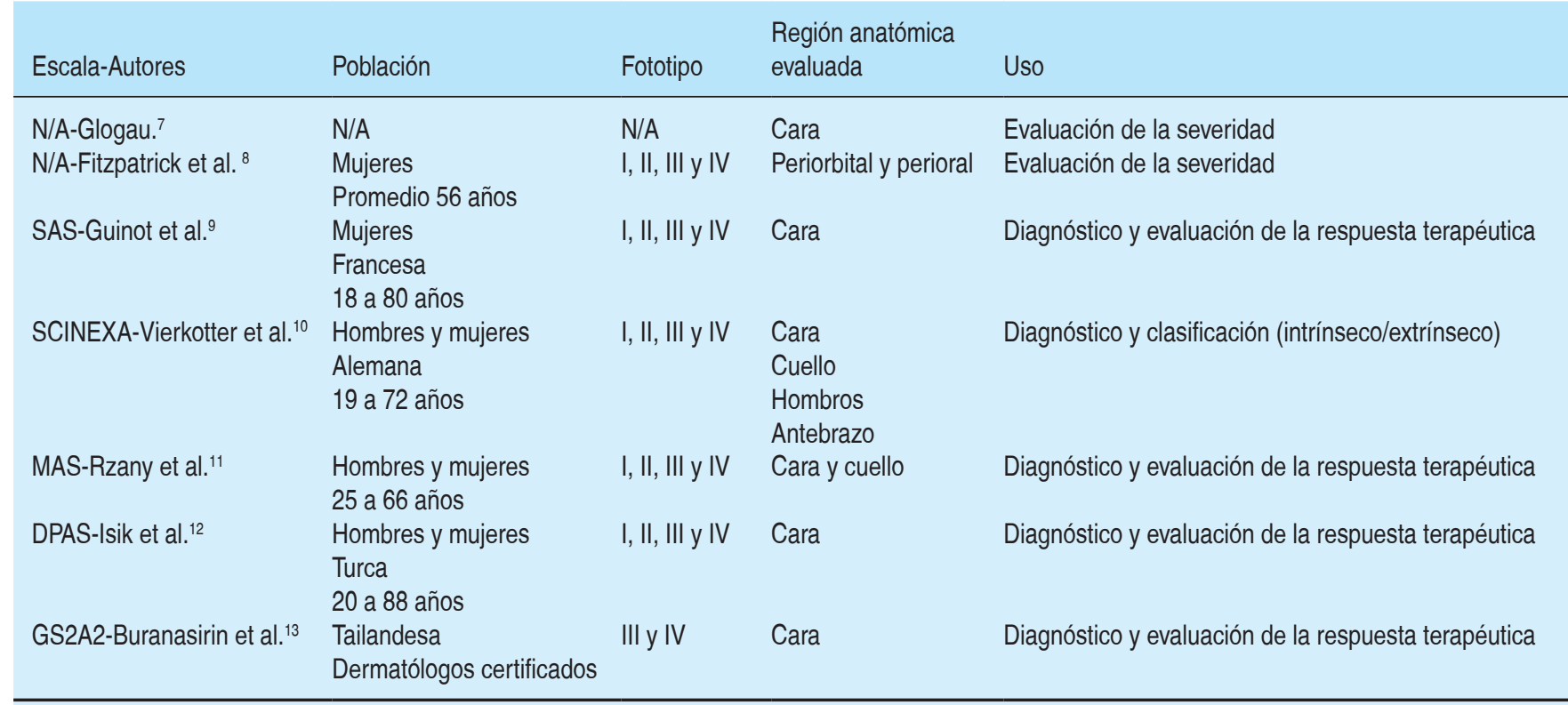

SAS = Skin Aging Score, SCINEXA = SCore of INtrinsic and EXtrinsic skin Aging, MAS = Merz Aesthetics Scale, DPAS = Dermoscopic Photoaging Scale, GS2A2 $=$ Global Subjective Skin Aging Assessment.

cara, cuello y cejas hace que se ocupe mucho tiempo para su llenado durante la práctica clínica.

$\mathrm{SAS}^{9}$ es una escala simple para valorar el envejecimiento. Engloba las características clínicas observadas en los pacientes en seis grupos de etiología común (comedones, quistes de milium, manchas hiperpigmentadas, arrugas, flacidez e incapacidad para enrojecer). Estudia áreas específicas como labios y región perioral; además de ser la única que toma en cuenta la presencia de comedones abiertos y cerrados. Se realiza evaluación global con puntaje entre 18 y 66 .

Por último, la escala fotonumérica de $\mathrm{Jdid}^{24}$ es la única que utiliza fotografías digitales de las diferentes zonas estudiadas, las cuales son analizadas en una interfaz computacional para optimizar la visualización de los signos clínicos y cuantificarlos con ayuda de un software, con lo que no se requiere de la opinión subjetiva del especialista.

En cuanto a la respuesta terapéutica, existe una escala que toma en particular consideración la percepción del paciente mediante el empleo de un diagrama de radar con escala Likert que evalúa 13 aspectos de la apariencia facial (pérdida de la elasticidad, rugosidad de la superficie, deshidratación, cicatrices, poros visibles, manchas hiperpigmentadas, manchas vasculares, arrugas dinámicas, arrugas estáticas, desequilibro del perfil, asimetría en vista frontal, flacidez y pérdida de volumen) y los agrupa en cuatro dominios: calidad de la piel, arrugas, morfología y volumen. En estos casos el paciente puede elegir la respuesta correcta durante su valoración y posterior al tratamiento prescrito. El puntaje de percepción oscila de cero a 39. Aunque se califica la percepción de los pacientes, no se ha realizado una correlación entre las escalas empleadas con un estándar para determinar si se relacionan la subjetividad y la objetividad del tratamiento con los datos descritos en la historia clínica. ${ }^{33}$

\section{Evaluación del envejecimiento de la piel extrafacial}

Se han elaborado unas cuantas escalas para calificar zonas especiales que miden la disminución del tejido graso y la laxitud de los tejidos blandos (flacidez), como en el cuello. ${ }^{30}$ Existen otras que analizan la severidad de las arrugas en el tórax anterior, tanto en reposo como en movimiento, ${ }^{31}$ así como la presencia de manchas hiperpigmentadas, ${ }^{21}$ algunas otras examinan datos de envejecimiento intrínseco en piel no fotoexpuesta de antebrazos: ${ }^{25}$ flacidez, líneas finas, xerosis y adelgazamiento de la piel; otras más el fotoenvejecimiento en brazos (arrugas, elastosis solar, lentigos solares, 
queratosis actínicas, leucodermia lenticular, pérdida de elasticidad y flacidez, púrpura y adelgazamiento de la piel). ${ }^{17}$

Por último, las manos ${ }^{19,32}$ son otra área extrafacial en la que los principales datos clínicos a calificar son la pérdida de volumen, la presencia de líneas muy finas, así como tendones y venas visibles.

Existe sólo una escala ${ }^{34}$ que analiza a nivel histopatológico el envejecimiento y fotoenvejecimiento mediante una biopsia de piel, lo cual la convierte en un instrumento objetivo y fiable. Sin embargo, al ser invasiva, costosa y debiendo ser realizada por personal calificado, se limita su uso en la consulta habitual, por lo que debería justificarse solo cuando la información que proporcionen las imágenes sea superior a los beneficios terapéuticos.

En la actualidad, no existe consenso sobre la valoración sistematizada del envejecimiento cutáneo debido a la gran cantidad de escalas existentes, ya que muchas de ellas examinan solamente ciertas regiones anatómicas (como el tercio superior, inferior o medio de la cara), son difíciles de aplicar por la cantidad y diversos valores que se califican en los ítems, y la dificultad para identificar adecuadamente los diferentes signos clínicos evaluados, como las alteraciones de la pigmentación, que pueden ser interpretadas de diferente forma por los especialistas. Sin embargo, escalas simples, rápidas y objetivas realizadas en pacientes con distintos fototipos permiten estimar el envejecimiento cutáneo y la respuesta terapéutica acorde a cada caso.

En este artículo se propone un algoritmo para la evaluación del envejecimiento (Anexo 1), el cual tiene como base los parámetros de las escalas ya existentes, con lo cual, se puede realizar un análisis más completo, objetivo, sistematizado y válido en fototipos semejantes a la población mexicana.

\section{CONCLUSIONES}

El estudio clínico del envejecimiento cutáneo debe incluir escalas que permitan una valoración objetiva y cuantificable, para realizar el diagnóstico y la evaluación de la respuesta terapéutica, como en las escalas GS2A2, DPAS y SAS. Incluir la búsqueda de datos tempranos de envejecimiento con ayuda de la dermatoscopía (debido a la limitación que el ojo humano tiene para percibir signos apenas visibles) podría prevenir el avance de factores modificables, como la exposición solar crónica o el hábito tabáquico, para así mejorar la respuesta terapéutica.
La escala de fotoenvejecimiento de Glogau y la de arrugas de Fitzpatrick utilizadas en la práctica clínica habitual han demostrado limitaciones subjetivas de interpretación y no permiten evaluar de manera objetiva la respuesta terapéutica. Las escalas como SCINEXA, SAS, DPAS y GS2A2 pueden ser utilizadas en poblaciones con fototipos III a $\mathrm{V}$, éstos incluyen a la población mexicana y admiten una valoración integral y sistematizada.

Hasta el momento no se han desarrollado ni validado escalas clínicas específicas en la población mexicana.

\section{REFERENCIAS}

1. Herrera-González NE, Aco-Flores AY. El melanoma en México. Rev Esp Med Quir. 2010; 15: 161-164.

2. Dobos G, Lichterfeld A, Blume-Peytavi U, Kottner J. Evaluation of skin ageing: A systematic review of clinical scales. Br J Dermatol. 2015; 172: 1249-1261. doi: 10.1111/bjd. 13509

3. Kohl E, Steinbauer J, Landthaler M, Szeimies RM. Skin ageing. J Eur Acad Dermatol Venereol. 2011; 25: 873-884. doi: 10.1111/j.14683083.2010.03963.x

4. Krutmann J, Bouloc A, Sore G, Bernard BA, Passeron T. The skin aging exposome. J Dermatol Sci. 2017; 85: 152-161. doi: 10.1016/j. jdermsci.2016.09.015

5. Baillie L, Askew D, Douglas N, Soyer HP. Strategies for assessing the degree of photodamage to skin: A systematic review of the literature. Br J Dermatol. 2011; 165: 735-742. doi: 10.1111/j.13652133.2011.10416.x

6. Sachs DL, Varani J, Chubb H, Fligiel SEG, Cui Y, Calderone K et al. Atrophic and hypertrophic photoaging: Clinical, histologic, and molecular features of 2 distinct phenotypes of photoaged skin. J Am Acad Dermatol. 2019; 81: 480-488. doi: 10.1016/j.jaad.2019.03.081

7. Glogau RG. Physiologic and structural changes associated with aging skin. Dermatol Clin. 1997; 15: 555-559. doi: 10.1016/s07338635(05)70465-4

8. Fitzpatrick RE, Goldman MP, Satur NM, Tope WD. Pulsed carbon dioxide laser resurfacing of photo-aged facial skin. Arch Dermatol. 1996; 132: 395-402. doi: 10.1001/archderm.1996.03890280047007

9. Guinot C, Malvy DJ-M, Ambroisine L, Latreille J, Mauger E, Tenenhaus $M$ et al. Relative contribution of intrinsic vs extrinsic factors to skin aging as determined by a validated skin age score. Arch Dermatol. 2002; 138: 1454-1460. doi: 10.1001/archderm.138.11.1454

10. Vierkotter A, Ranft U, Kramer U, Sugiri D, Reimann V, Krutmann J. The SCINEXA: A novel, validated score to simultaneously assess and differentiate between intrinsic and extrinsic skin ageing. J Dermatol Sci. 2009; 53: 207-211. doi: 10.1016/j.jdermsci.2008.10.001

11. Rzany B, Carruthers A, Carruthers J, Flynn TC, Geister TL, Gortelmeyer R et al. Validated composite assessment scales for the global face. Dermatol Surg. 2012; 38: 294-308. doi: 10.1111/j.15244725.2011.02252.x

12. Isik B, Gurel MS, Erdemir AT, Kesmezacar O. Development of skin aging scale by using dermoscopy. Skin Res Technol. 2013; 19: 69-74. doi: $10.1111 /$ st. 12033 
13. Buranasirin P, Pongpirul K, Meephansan J. Development of a Global Subjective Skin Aging Assessment score from the perspective of dermatologists. BMC Res Notes. 2019; 12: 364. doi: 10.1186/s13104019-4404-z.4

14. Terwee CB, Mokkink LB, Knol DL, Ostelo RWJG, Bouter LM, de Vet HCW. Rating the methodological quality in systematic reviews of studies on measurement properties: a scoring system for the COSMIN checklist. Qual Life Res. 2012; 21: 651-657. doi: 10.1007/ s11136-011-9960-1

15. Narins RS, Carruthers J, Flynn TC, Geister TL, Gortelmeyer R, Hardas $B$ et al. Validated assessment scales for the lower face. Dermatol Surg. 2012; 38: 333-342. doi: 10.1111/j.1524-4725.2011.02247.x

16. Luebberding S, Krueger N, Kerscher M. Comparison of validated assessment scales and $3 \mathrm{D}$ digital fringe projection method to assess lifetime development of wrinkles in men. Skin Res Technol. 2014; 20: 30-36. doi: 10.1111/srt.12079

17. Guimaraes COZ, Bagatin E, Guadanhim LRS, Sternberg F, Picosse FR, Nunes $\mathrm{G}$ et al. Development and validation of a clinical scale for the evaluation of forearm skin photoaging. J Cutan Med Surg. 2015; 19: 380-387. doi: 10.1177/1203475415574946

18. Cohen JL, Thomas J, Paradkar D, Rotunda A, Walker PS, Beddingfield FC et al. An interrater and intrarater reliability study of 3 photographic scales for the classification of perioral aesthetic features. Dermatol Surg. 2014; 40: 663-670. doi: 10.1111/dsu.0000000000000008

19. Cohen JL, Carruthers A, Jones DH, Narurkar VA, Wong M, Cheskin $\mathrm{LN}$ et al. A randomized, blinded study to validate the Merz Hand Grading Scale for use in live assessments. Dermatol Surg. 2015; 41: S384-388. doi: 10.1097/DSS.0000000000000553

20. Leal Silva HG. Facial laxity rating scale validation study. Dermatol Surg. 2016; 42: 1370-1379. doi: 10.1097/DSS.0000000000000915

21. Landau M, Geister TL, Leibou L, Blessmann-Gurk B, Gortelmeyer $\mathrm{R}$, Frand $\mathrm{J}$ et al. Validated assessment scales for décolleté wrinkling and pigmentation. Dermatol Surg. 2016; 42: 842-852. doi: 10.1097/ DSS.0000000000000786

22. Sen S, Choudhury S, Gangopadhyay A, Halder C, Biswas P, Jain A. A clinical rating scale for the assessment of facial aging in Indian population. Indian J Dermatol Venereol Leprol. 2016; 82: 151-161. doi: 10.4103/0378-6323.174369

23. Carruthers J, Donofrio L, Hardas B, Murphy DK, Jones D, Carruthers A et al. Development and validation of a photonumeric scale for evaluation of facial fine lines. Dermatol Surg. 2016; 42: S227-234. doi: 10.1097/DSS.0000000000000847

24. Jdid R, Latreille J, Soppelsa F, Tschachler E, Morizot F. Validation of digital photographic reference scales for evaluating facial aging signs. Skin Res Technol. 2018; 24: 196-202. doi: 10.1111/ srt. 12413
25. Chien AL, Qi J, Grandhi R, Harris-Tryon T, Kim N, Jang MS et al. Chronological aging in African-American skin: a reliable photonumeric scale demonstrates age and body mass index as contributing factors. J Natl Med Assoc. 2018; 110: 534-539. doi: 10.1016/j.jnma.2018.01.005

26. La Padula S, Hersant B, Bompy L, Meningaud JP. In search of a universal and objective method to assess facial aging: the new face objective photo-numerical assessment scale. J Craniomaxillofac Surg. 2019; 47: 1209-1215. doi: 10.1016/j.jcms.2019.03.014

27. Flynn TC, Carruthers A, Carruthers J, Geister TL, Gortelmeyer R, Hardas B et al. Validated assessment scales for the upper face. Dermatol Surg. 2012; 38: 309-319. doi: 10.1111/j.15244725.2011.02248.x

28. Carruthers J, Flynn TC, Geister TL, Gortelmeyer R, Hardas B, Himmrich $S$ et al. Validated assessment scales for the mid face. Dermatol Surg. 2012; 38: 320-332. doi: 10.1111/j.1524-4725.2011.02251.x

29. Tsukahara K, Takema Y, Fujimura T, Moriwaki S, Kitahara T, Imokawa G. Determination of age-related changes in the morphological structure (sagging) of the human cheek using a photonumeric scale and three-dimensional surface parameters. Int J Cosmet Sci. 2000; 22: 247-258. doi: 10.1046/j.1467-2494.2000.00033.x

30. Sattler G, Carruthers A, Carruthers J, Flynn TC, Geister TL, Gortelmeyer R et al. Validated assessment scale for neck volumen. Dermatol Surg. 2012; 38: 343-350. doi: 10.1111/j.15244725.2011.02253.x

31. Fabi S, Bolton J, Goldman MP, Guiha I. The fabi-bolton chest wrinkle scale: a pilot validation study. J Cosmet Dermatol. 2012; 11:229-234. doi: 10.1111/j.1473-2165.2012.00628.x

32. Lee JH, Choi YS, Park ES, Kim JS, Kang MS, Oh HY et al. A novel photonumeric hand grading scale for hand rejuvenation. Arch Plast Surg. 2019; 46: 359-364. doi: 10.5999/aps.2019.00521

33. Jain R, Huang P, Ferraz RM. A new tool to improve delivery of patientengaged care and satisfaction in facial treatments: the aesthetic global ranking scale. J Cosmet Dermatol. 2017; 16: 132-143. doi: 10.1111/ jocd. 12297

34. Kawabata K, Kobayashi M, Kusaka-Kikushima A, Akasaka E, Mabuchi T, Fukui T et al. A new objective histological scale for studying human photoaged skin. Ski Res Technol. 2014; 20: 155-163. doi: 10.1111/ srt.12099

Correspondencia:

Dra. Viridiana Vera Ramírez

Juárez Norte 203, tercer piso,

Col. Centro, 43600,

Tulancingo de Bravo, Hidalgo.

E-mail: virivera.derma@gmail.com 
Anexo 1: Ejemplo de algoritmo de evaluación de envejecimiento de la piel y escalas recomendadas.

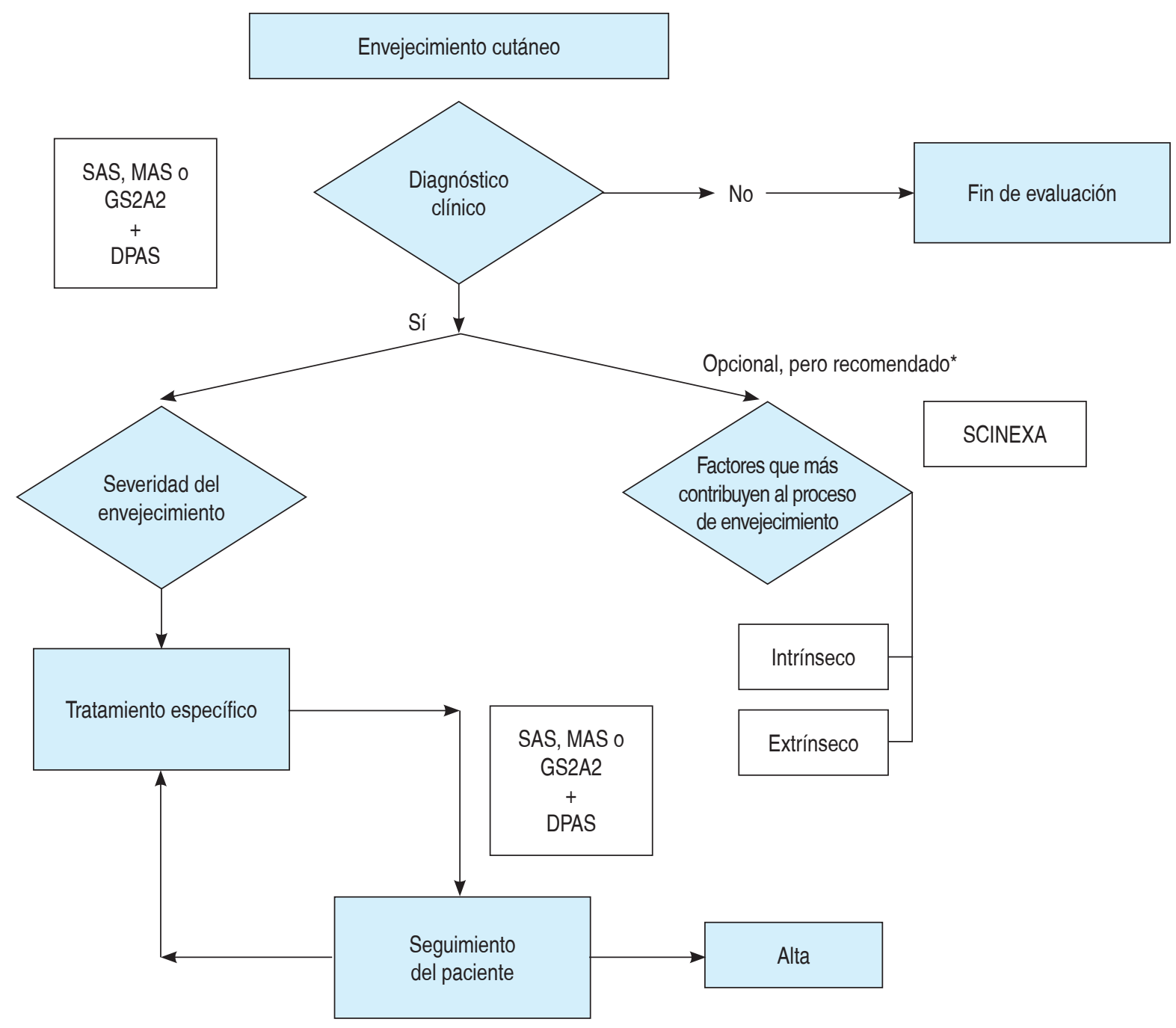

*Aunque la evaluación del envejecimiento de la piel (intrínseco vs extrínseco) no es imprescindible para el diagnóstico, es recomendable su realización, ya que permite adecuar el tratamiento a la etiología.

SAS = Skin Aging Score, MAS = Merz Aesthetics Scales, GS2A2 = Global Subjective Skin Aging Assessment, DPAS = Dermoscopic Photoaging Scale, SCINEXA = SCore of INtrinsic and EXtrinsic skin Aging. 